\title{
Effects of Intensity Method on MEF of Biceps Brachii and Number of Repetition during Barbell Curl Exercise
}

\author{
Kim, Ki-Hong, Lee, Il-Suk, Jung, Song-Young,Yang, Chun-Ho
}

\begin{abstract}
The purpose of this study was to investigate the number of repetitions and the MEF of the Biceps brachii for the 3 sets of maximal repetitive barbell curls with 3intensity. The subjects which were selected as men in their 20s who had more than 2 years of resistance exercise experience. The subjects also conducted a bar-cull experiment at each 7-day interval with a randoml assignment (50\%1RM, 70\%1RM, 90\%1RM). In every one of the sets, the number of repetitions and the MEF of the Biceps brachii were measured. The measured two-way ANOVA was used, and the statistical significance was noted at the result of $a=.05$. The higher the intensity condition, the fewer the number of repetitions performed by the subject. The decrease of the number of repetitions was larger with a lower intensity condition. The lower the intensity, the higher the level of muscle fatigue was noted in the subject. In conclusion, it seems that the maximum repetitive exercise is seen to increase the muscle fatigue as the intensity is lower in those instances. Therefore, it is necessary to write a program considering the degree of muscle fatigue at low intensity in resistance exercise for the best results during an exercise program.
\end{abstract}

Keywords :Repetition, MEF, Muscle fatigue, Barbell Curl, Resistance training.

\section{INTRODUCTION}

$\mathrm{R}$ nerve roots, and it is aimed at hypertrophy and strengthening of the muscles [1]. Various resistance exercises are carried out for the benefit of an elite athlete's health management, improvement of performance and injury prevention, and it has a positive effect on the ultimate improvement of muscular strength such as increasing endurance, and muscular strength $[2,3]$. These forms of exercise are also effective for rehabilitation and pain management to recover from injuries [4].The biceps brachii is the muscle that many people want to develop first when initiating a workout regimen, and the muscle functions to bend the elbow when lifting the forearm inward toward the arm. To begin with, a barbell curl is a typical biceps brachii exercise that affects the biceps brachii and brachialis, and it also exerts a load on the brachioradialis,

Revised Manuscript Received on July 22, 2019.

* Correspondence Author

Kim, Ki-Hong, Dankook University, Dept. of Recreation and Leisure studies, Cheonan,31116, Korea

Lee, Il-Suk, Dankook University, Dept. of General Education, Cheonan,31116, Korea

Jung, Song-Young, Dankook University, Dept. of Physical Education, Yongin, 16979, Korea

Yang, Chun-Ho, Hanseo University, Dept. of Marine Sports, Seosan,31962, Korea

flexor carpi ulnaris and flexors of the fingers and cuffs[5]. In order to achieve the goal of exercise through resistance exercise, individualized programs are needed and should be matched according to an individual's ability level and condition. And according to the goal of training, factors such as exercise load, break time, repetition frequency, set number, should also be considered and combined to create a safe workout schedule [6].In general, resistance exercise can be divided into muscle endurance type, hypertrophy type and muscle strength type. It is recommended that all sets of exercises utilized to work out the muscles are performed to the maximum number of repetitions possible, also known as the maximum repetition (RM). This program of exercise should be performed for each performance objective [7]. Depending on the purpose of the exercise, the resistance exercise of muscle endurance type should be more than 12 repetitions that are performed at an intensity of less than $67 \%$ of $1 \mathrm{rm}$. The hypertrophy type resistance exercise is performed with 6 to 12 repetitions with an intensity of $67-85 \% 1 \mathrm{RM}$, and the muscle strength exercise can be performed with less than 6 repetitions with intensity of $85 \%$ or more of $1 \mathrm{RM}[8,9]$. In this relation, it is important to use a rest interval, which is recommended differently depending on the purpose of exercise [4]. Previous studies on the proper resting time for the performance of an exercise vary. In general, muscle-endurance type resistance exercise is known to be less than 1 minute, hypertrophy type resistance exercise is between 1-3 minutes, and muscle strength type resistance exercise is between 3-5 minutes [3]. Studies concerning the intensity in regard to improving strength by resistance exercises claim that 2 to 6 sets of resistance exercise improved strength both in experts and unskilled participants [10-14], and additionally reported that there was no difference in strength improvement between 2 sets and 3 sets, between 2 sets and 4 sets, but more than 3 sets of exercise showed better strength improvement than just performing 1 set. Therefore, the set should be varied according to the exercise goal. In resistance exercise, three to six sets are generally used, but more or less sets are seen to be effective in improving overall muscle strength[6]. Resistance exercise is known to be a factor and induces fatigue, which generally increases as the load increases, and the higher the rate of fast twitch muscle fibers, the easier fatigue appears [15]. Excess muscle fatigue induces stress rather than physiological adaptation, which reduces overall muscular function and exercise performance[4]. As the muscle is

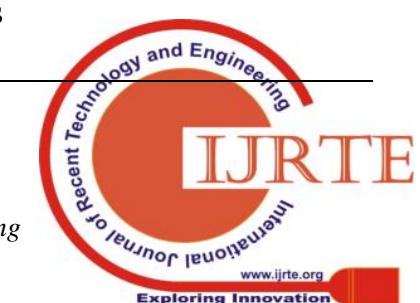


repeatedly exercised, ATP (adenosine triphosphate) decreases with the onset of chemical changes, whereby the ILactic acid and wastes are built up in the muscle, and the contraction frequency of the exercise unit is decreased, there by affecting exercise performance. The decreasing contraction frequency can be quantified by a review and frequency analysis of the measured EMG signal. It can be concluded that fatigue of muscle is accumulated as the tendency of the frequency distribution analyzed in one muscle is lowered [16]. As muscle fatigue accumulates, the frequency of muscle activity decreases. Broadly speaking, when the EMG signal measured in the fatigued muscle is changed to the frequency band, the mean frequency or median frequency of the frequency is lower than that measured in the non-fatigued muscle $[17,18]$. In other words, the intermediate frequencies in muscles not fully restored from fatigue are reduced more rapidly than normal muscles. Changes in muscle fatigue affected the reduction in the ability to exercise and maintain muscle strength. It is also noted that there is a decrease in the action potential conduction velocity along the muscle fiber and consequently a change in the shape of the power spectrum. In this study, we investigated the reduction of median frequency of the repetitions and biceps brachii power spectrum in 3 set repetition maximum exercises. The purpose of this study is to investigate the basic data that can be used to create the maximum repetitive exercise program.

\section{STUDY METHODS}

After selecting the subjects, it was noted that the 1RM of the barbell curls was measured to pre-test the data. After 7 days rest period for the experiment after the pre - test, the subjects were selected through the randomly assignment of three exercise load conditions (50\%1RM, 70\%1RM, $90 \% 1 \mathrm{RM}$ ) according to the resistance exercise intensity and then the experiment was conducted. In this experiment, the number of repetitions and the EMG of the biceps brachii were measured. In the meantime, the experiments were conducted at intervals of one week to perform all weight loading conditions. The experimental design of this study is shown in "Figure 1", and the exercise program used in the experiment is shown in "Figure 2".

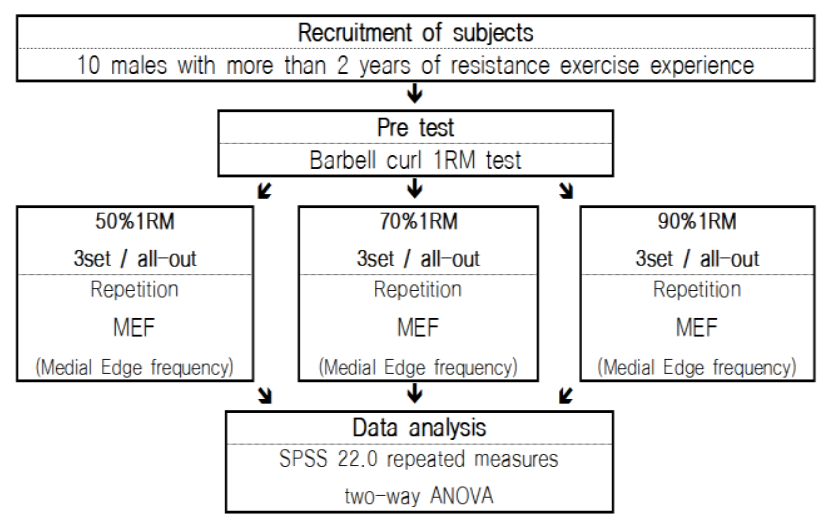

Figure 1. Experimental Design

\begin{tabular}{|ccccc|}
\hline Intensity & $\begin{array}{c}\text { Rest } \\
\text { interval }\end{array}$ & 1set & 2set & 3set \\
\hline $50 \% 1 \mathrm{RM}$ & $1 \mathrm{~min}$ & All out & All out & All out \\
\hline $70 \% 1 \mathrm{RM}$ & $2 \mathrm{~min}$ & All out & All out & All out \\
\hline $90 \% 1 \mathrm{RM}$ & $3 \mathrm{~min}$ & All out & All out & All out \\
\hline
\end{tabular}

Figure 2. Exercise program

\subsection{Subjects}

To begin with, the subjects of this study were 10 male students in their twenties who had more than 2 years of resistance exercise experience without any physical illness, among the students at D university in Cheonan city. The subjects in this case were fully informed about the purpose and procedure of the pre-experiment study, and only those who voluntarily participated in the study were selected. The physical characteristics of the subjects are shown in "Table 1 ".

Table 1: Characteristics of the subject

\begin{tabular}{|c|c|c|c|}
\hline & Age(year) & Weight $(\mathrm{kg})$ & Height $(\mathrm{cm})$ \\
\hline $\mathrm{N}=10$ & $27.80 \pm 2.57$ & $82.00 \pm 9.02$ & $174.90 \pm 3.72$ \\
\hline
\end{tabular}

\subsection{RM measurement}

In this study, we measured the barrel curl $1 \mathrm{RM}$ of the subjects more safely by the direct measurement method [4] based on the indirect measurement method [19].

\section{$1 \mathrm{RM}$ indirect measurement}

The subjects were asked to select the appropriate weight to lift about 7-8 reps and then to select the weight to lift. At that time, the 1RM was estimated by substituting the lifted weight into the equation below.

The weight that can be lifted more than 10 reps is likely to cause an error in the formulation of the 1RM calculation. Therefore, it was conducted at 10 reps or less.

$$
1 \mathrm{RM}=\mathrm{W} 0+\mathrm{W} 1
$$

$\mathrm{W} 0=$ After enough warm-up, 7-8 reps load that can be repeatedly contraction.

$$
\mathrm{W} 1=\mathrm{W} 0 \times 0.025 \times \mathrm{R}
$$

$$
\mathrm{R}=\text { Number of repetitions }
$$

\section{$1 \mathrm{RM}$ direct measurement}

Based on the 1RM estimated by the indirect measurement method, at this time the 1RM was measured directly, and the method of the National Strength and Conditioning Association (NSCA) was used [4]

\subsection{Exercise Method}

The position of the barbell curl was performed with reference to the method of [4]. The starting position was the position of the barbell at the front of the femur and the position of the shoulder-width underhand grip. The width of the posterior leg was shoulder width, in order that the knee was in a position to be slightly bent. During the performance, the elbow was flexed until the bar was positioned in front of the anterior deltoid, and the

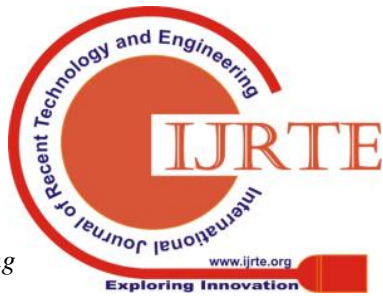


back was tilted to the back, or the back was leaned against the wall to prevent the recursion from being performed. The elbows were fixed using an instrument (Arm blasster).

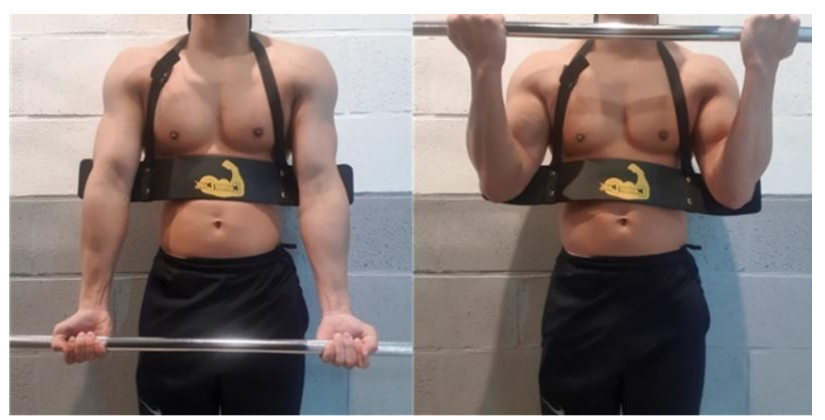

Figure 3. Barbell curl exercise

\subsection{EMG measurement}

The muscle activity was measured using an electromyography machine (LXM 5308, Korea). In this experiment, two surface electrodes were attached at $1 \mathrm{~cm}$ apart from the insertion site of the muscular electrodes. The location of the electrodes was attached to the middle bundle of the upper arms of the biceps brachii. It is important to note, that to obtain good EMG data, subjects were asked to use a razor. The skin of the skin outer layer was shaved and wiped with an alcohol cotton to clean the skin surface. The equipment required for all EMG measurements was unified and attached to the same experimenter. The wires connecting the electrode and the electromyogram before this measurement were assembled and fixed with tape, and subsequently, the noise was minimized by measurement. The channel of the electrode attachment region was designated by using Telescan software during the measurement and then monitored at that time. In this relation, the surface electrodes attached site-specific muscle is shown in "Figure 4".

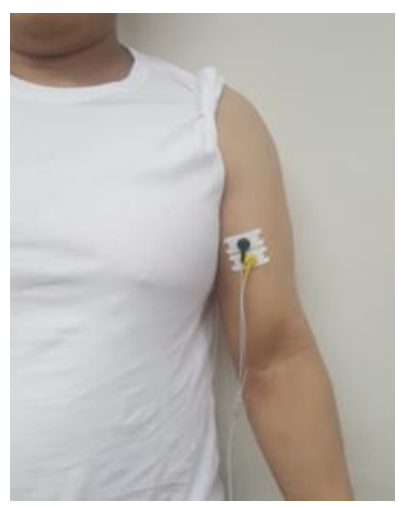

Figure 4. Location of electrode

\subsection{Data processing}

Data processing of the present study, we used a SPSS 22.0 statistical program, and thus all variables were calculated with the mean and standard deviation. When the movement load 3 with a set (3) the maximum iteration was used repeated measures two - way ANOVA method to analyze the data obtained during exercise, a significant difference in the receive to the post compared to contrast the repeated methods were applied. In this case, the statistical significance was set at $\alpha=.05$.

\section{RESUITS}

\subsection{Variation in the number of repeats}

"Table 2" shows the results of repeated measures two-way ANOVA for the number of repetitions in the maximum repetitive motion of the barbell curl according to the exercise load and set. Evidently, there was a significant difference in repetition frequency according to the exercise load, and it was higher in order of 50\% 1RM, 70\% 1RM, 90\% $1 \mathrm{RM}(\mathrm{p}=.001)$. There was a significant difference in the number of repetitions according to the set. In 50\% 1RM and $70 \% 1 \mathrm{RM}$, the number of repetitions was decreased gradually from 1 set to 3 sets. In 90\% 1RM, 1 set was higher than 2 sets and 3 sets. Therefore, in this case, there was a significant difference $(p=.001)$ between the effect of the exercise load and the number of repetitions according to the set

Table 2: Repeated measurements two-way ANOVA results for the number of repetitions

\begin{tabular}{|c|c|c|c|c|c|}
\hline Intensity & 1 set & 2 set & 3 set & source & F \\
\hline $50 \% 1 \mathrm{RM}$ & $26.2 \pm 6.20$ & $10 \pm 2.19$ & $6.5 \pm 2.27$ & Intensity(I) & 87.939 \\
\hline $70 \% 1 \mathrm{RM}$ & $12 \pm 7.10$ & $7.1 \pm 1.29$ & $4.5 \pm 1.27$ & Sets(S) & 215.282 \\
\hline $90 \% 1 \mathrm{RM}$ & $3.4 \pm 0.84$ & $2.4 \pm 0.70$ & $2 \pm 0.82$ & (I) $\times(\mathrm{S})$ & 68.454 \\
\hline \multicolumn{6}{|l|}{$\mathrm{M} \pm \mathrm{SD}$} \\
\hline \multicolumn{6}{|l|}{ Reps } \\
\hline \multicolumn{6}{|l|}{30} \\
\hline \multicolumn{6}{|l|}{25} \\
\hline \multicolumn{6}{|r|}{$-50 \% 1 \mathrm{RM}$} \\
\hline \multicolumn{6}{|r|}{$\leftarrow 70 \% 1 \mathrm{RM}$} \\
\hline \multicolumn{2}{|c|}{ 然 } & \multicolumn{4}{|r|}{$-90 \% 1 \mathrm{RM}$} \\
\hline \multirow[t]{2}{*}{0} & & & & & \\
\hline & 1set & & & 3set & \\
\hline
\end{tabular}

Figure 5. Change in the number of repetition

\subsection{Biceps brachii MEF changes}

"Table 3" shows the repeated measures two-way ANOVA for the MEF changes in the biceps brachii of the maximal repetitive motion of the barbell curl, according to the exercise load and set. The prevailing discipline notes that there was a significant difference in the MEFs of the upper arm and lower extremities according to the exercise load ( $\mathrm{p}=$ .001 ) in the order of 50\% 1RM, 70\% 1RM and 90\% 1RM. Additionally, it is noted that there was no significant difference in the MEF according to the set, and there was no significant difference in the interaction effect between the exercise load and the repetition frequency according to the set (p <.005).

Table 3: Repeated measurements two-way ANOVA results for $\mathrm{MEF}$ changes $(\mathrm{Hz})$

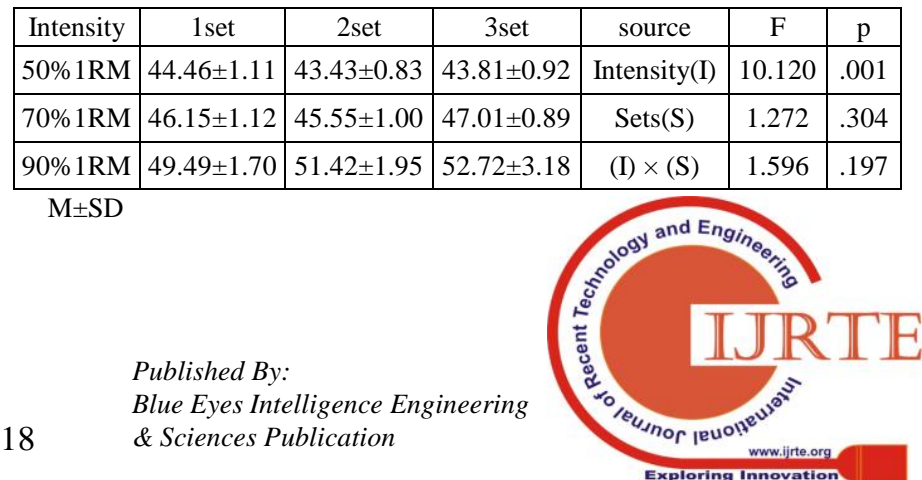




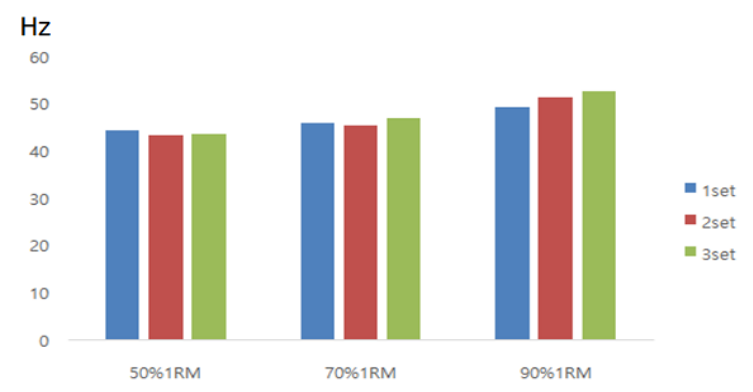

Figure 6. Change in the MEF

\section{DISCUSSION}

The acute response and chronic adaptation of resistance exercise can vary depending on weight intensity and exercise volume[4]., Depending on the purpose of exercise, 50\% 1RM is recommended as muscle endurance, $70 \% 1 \mathrm{RM}$ is noted at muscle hypertrophy, and $90 \% 1 \mathrm{RM}$ is the training intensity for maximum muscle strength[3]. In this case, the exercise volume is the product of the weight intensity and the number of repetitions. The number of repetitions according to the load intensity decrease with the weight, and the lighter the weight, it is noted that the higher the amount of exercise occurs due to the number of repetition can be performed[9]. In this study, the number of repetitions tends to decrease as the set progresses, and the number of repetitions is high in the order of 50\% 1RM, 70\% 1RM, and 90\% 1RM. In addition, as exercise intensity decreased, exercise time increased due to increase of repetition frequency, Therefore, it seems to be verified that the number of repetition times decreased rapidly as the set of the creatine phosphate and ATP decreased in the body[16]. When resistance exercise is performed with low exercise intensity, mobilization of the slow-twitch starts first, and when the fatigue process starts to occur, the fast-twitch is mobilized to generate force during the exercise. Finally, when all of the muscle fibers are exhausted, the exercise of the set is finished without generating any more force[20,21]. Considering the rest time between the exercise sets, a short rest is required due to the oxidative characteristic of the slow-twitch. On the other hand, the fast-twitch have a stronger glycolysis characteristic and require a longer rest time[22]. Because in the instance when the fast-twitch generates energy through the aerobic process, it is because of a high level of lactic acid that is known to have accumulated during high intensity exercise. In the usual case, the accumulation of lactic acid lowers the intracellular $\mathrm{pH}$ through the decomposition of hydrogen ion $(\mathrm{H}+)$, which eventually leads to muscle fatigue[23, 24]. In this caes, it is Komi \&Tesch [25] suggested that $50 \%$ for $20 \mathrm{sec}, 75 \%$ for $40 \mathrm{sec}, 87.5 \%$ for $60 \mathrm{sec}$, and $100 \%$ for 3 min for recovery of ATP and PC is necessary to determine fatigue. In this study, the decrease in the number of repetitions tended to decrease as the rest interval between sets increased. As the set continued, it seemed that long rest were needed for many repetition[26]. It is also reported that exercise was reduced when the resistance exercise was applied with a short resting time, Arabadzhiev, Dimitrov \& Dimitrova [27] and concluded that the resistance exercise using a long resting time reduced the decrease in exercise volume between the sets. Also Konred[28] this is a time to recharge more than 3 minutes of ATP in the body. In Medina $\&$ Badillo [16], it was shown that the creatine phosphate (PCr) stored in the muscle during exercise is reduced by $40 \%$ within 10 seconds, and by more than $50 \%$ within 20 seconds. It is noted that it takes 7 minutes for a muscle to approach the recovery of $95 \%$ respectively. In this study, it was verified that the total work reduction rate was the lowest in $90 \%$ strength, with 3 minutes rest period in the maximum repetitive exercise according to the exercise load. This suggests that restoration of the creatine phosphate in the body is the most adequate, taking more rest than the $50 \%$ intensity and the $70 \%$ intensity noted during the study.

The purpose of this study was to investigate the incidence of muscle fatigue of the biceps brachii during the barbell curl exercise, by using the surface electromyography is an objective measurement, that can quantify the degree of muscle contraction caused by the action of muscles and nerves[29]. By measuring the complex movement of the muscles under the electrode, is possible to identify the activity of a particular muscle[30]. By the same token, the frequency spectrum analysis is based on the conversion of EMG signals to frequency planes for a certain period of time. It is believed that the influence of electrodes is small and the changes are closely related to the conduction velocity of muscles and the characteristics of action potentials of exercise units., It is an effective way to electrophysiologically detect local fatigue, and can estimate a fine point of muscle locus[31].The central frequency, the midpoint of the frequency spectrum, can tell the difference in fatigue levels in the muscles with frequencies showing the maximum amplitude, as seen by analyzing the activity potential variation of the muscles in the fast Fourier transformations (FFT) method. In this study, MEF decreased in 2 sets after 1 set at 50\% 1RM and 70\% 1RM, and MEF increased at the 3 set. For $90 \%$ 1RM, the MEF also increased as the set progressed at $50 \% 1 \mathrm{RM}$, and $70 \%$ at $1 \mathrm{RM}$., The reduction of MEFs in the two sets after one set is to maintain the exercise volume in the absence of muscle fatigue elements and energy substrates in order to verify that the Type 1 muscle fibers seemed to have demonstrated muscle strength. The increase of MEF in 3 sets seems to be attributed to the low number of repetitions due to the measured short-term muscle strength of the fast twitch [15]. In addition, the increase of MEF according to the set at $90 \%$ load is considered to be the recovery of fatigue substances, such as $\mathrm{pH}$ and lactic acid, due to the long resting time compared to other exercise intensity conditions (50\% 1RM / 1 minute rest, $70 \%$ 1RM /2 minutes rest). In general, when there is fatigue in the neuromuscular, the transmission rate of the active potential is slowed down, and the measured frequency is consequently lowered. In the absence of fatigue, the frequency is transmitted at a high speed. Hultman [31], whereas it is reported that the frequency of the stimulation of the skeletal muscles is $30-200 \mathrm{~Hz}$.

In general, it is seen that the peak point of maximal contraction is located between $100-200 \mathrm{~Hz}$, and it falls rapidly and becomes 0 at about $80 \mathrm{~Hz}$. In addition, the motor units of high-fatigue fast twitch are fatigued and the nerve conduction velocity is lowered, 
considering that the nerve firing is no longer considered to be carried out, and only the remaining slow twitch nerve firing is the result of this effort. As the results of this case study data[29], it is understood that the incidence of a slow twitch moves slowly from the high frequency to the low frequency, because the nerve conduction velocity is slow and the MEF is low[32]. It is noted that mobilized fast twitch can occur after the mobilization of a slow twitch, and muscle fatigue is easy to occur at that time, due to the increased use of fast twitch. According to the results, the lower the load in the barbell curl according to the load, the higher the MEF and the recurring repetitions. It seems that muscle fatigue is proportional to exercise volume, and it is considered that less muscle volume occurs at low fatigue. However, it is important to note that low muscle fatigue levels in heavy loads seem to prevent the exercise from continuing, before the acidification process develops in the body, whereas at that time more sets of exercise can be sustained. This supports the general theory that the higher the total measured exercises volume, the higher the degree of muscle fatigue that will be experienced by performing those exercises.

\section{CONCLUSION}

The results showed that there was a significant difference in the number of repetitions according to exercise load, and $50 \%$ 1RM, 70\% 1RM, and 90\% 1RM were higher ( $\mathrm{p}=.000)$. There was a significant difference in the number of repetitions according to the set $(\mathrm{p}=.000)$. In 50\% $1 \mathrm{RM}$ and 70\% 1RM, in relation to the data confirming that the set was reduced gradually as the set from 1 set to 3 sets proceeded. In $90 \%$ 1RM, 1 set was higher than 2 sets and 3 sets $(p=.000)$. There was a significant difference noted in the interaction effect between the exercise load, and the number of repetitions according to the set. As was expected, there was a significant difference in the MEF changes of biceps brachii according to the exercise load in the order of 50\% 1RM, 70\% 1RM and $90 \%$ 1RM. As a result, the number of repetitions decreased with increasing intensity, and it was shown that the MEF increased with increasing intensity. This means that as the number of repetitions increases, it is noted that muscle fatigue increases, while as the intensity decreases, at that time the muscle fatigue increases. Therefore, it is considered that muscle fatigue in low-intensity high-repetition should be reviewed and analyzed when writing and creating a new and effective resistance exercise program.

\section{REFERENCES}

1. K.H.Kim, W.Y. Park, J.S. Kim, "The Effect of Changing Intensity Between Sets on Agonist Muscle Activity, iEMG, MEF, CK After Bench Press". The Korean Journal of Physical Education. 51(2), 379-387, 2012.

2. W.J. Kraemer, N.A. Ratamess, "Physiology of resistance training: current issues". Orthopaedic Physical Therapy Clinics of North America. 9(4), 467-514, 2000.

3. T.O. Bompa, C. Buzzichelli, Periodization-: theory and methodology of training. Human Kinetics, 2018.

4. G.G. Haff, N.T. Triplett, Essentials of strength training and conditioning 4th edition. Human kinetics, 2015.

5. W.J. Kraemer, N.A. Ratamess, "Fundamentals of resistance training: progression and exercise prescription". Med Sci Sports Exerc. 36(4), 674-88, 2004.

6. M. Izquierdo, J.J. Gonza'lez-Badillo, K. Ha"kkinen, et al, "Effect of loading on unintentional lifting velocity declines during single sets of repetitions to failure during upper and lower extremity muscle actions". Int J Sports Med. 27(9), 718-24, 2006.

7. N. Madsen, T. McLaughlin, "Kinematic factors influencing performance and injury risk in the bench press exercise". Medicine and science in sports and exercise. 16(4), 376-381, 1984.

8. T. Lawton, J. Cronin, E. Drinkwater, R. Lindsell, D. Pyne, "The effect of continuous repetition training and intra-set rest training on bench press strength and power". J Sports Med Phys Fitness. 44(4), 361-367, 2004.

9. A.D. Walshe, G.J. Wilson, G.J. Ettema, "Stretch-shorten cycle compared with isometric preload: contributions to enhanced muscular performance". Journal of Applied Physiology. 84(1), 97-106, 1998.

10. R.A. Conwit, D. Stashuk, H. Suzuki, N. Lynch, M. Schrager, et al, "Fatigue effects on motor unit activity during submaximal contractions". Archives of physical medicine and rehabilitation. 81(9), 1211-1216, 2000.

11. J. Elliott, B.A. Callingham, D.F. Sharman, "The influence of amine metabolizing enzymes on the pharmacology of tyramine in the isolated perfused mesenteric arterial bed of the rat". British journal of pharmacology. 98(2), 515-522, 1989.

12. R. Van Den Tillaar, G. Ettema, "The sticking period in a maximum bench press". Journal of sports sciences. 28(5), 529-535, 2010.

13. R.M. Enoka, D.G. Stuart, "Neurobiology of muscle fatigue". J Appl Physiol. 72(5), 1631-48, 1992.

14. C. Williams, S. Ratel, Human muscle fatigue. Routledge, 2009.

15. D.A. Jones, "Changes in the force-velocity relationship of fatigued muscle: implications for power production and possible causes". J Physiol. 588(16), 2977-86, 2010.

16. L. Sanchez-Medina, J.J. González-Badillo, "Velocity loss as an indicator of neuromuscular fatigue during resistance training. Medicine and science in sports and exercise". 43(9), 1725-1734, 2011.

17. N.A. Evans, Bodybuilding anatomy. Human Kinetics, 2018.

18. P.A. Davidson, M. Pink, J. Perry, et al, "Functional anatomy of the flexor pronator muscle group in relation to the medial collateral ligament of the elbow". The American journal of sports medicine. 23(2), 245-250, 1995 .

19. G.W. Woods, H.S. Tullos, "Elbow instability and medial epicondyle fractures". The American journal of sports medicine. 5(1), 23-30, 1977.

20. S.K. Lim, "Review of ulnr collateral ligament injuries and prevention in baseball players". Journal of coaching development. 9(3), 65-80, 2007.

21. J.M. Willardson, L.N. Burdett, "The effect of rest interval length on bench press performance with heavy vs. light loads". J Strength Cond Res. 20, 396-399, 2006.

22. J.T. Viitasalo, P.W. Komi, "Interrelationships of EMG signal characteristics at different levels of muscle tension and during fatigue". ElectromyogrClinNeurophysiol. 18(3-4), 167-78, 1987.

23. P.V. Komi, M. Kaneko, O. Aura, "EMG activity off the leg extensor muscles with special reference to mechanical efficiency in concentric and eccentric exercise". International Journal of Sports Medicine suppl. 1, 22-9, 1987

24. T. Moritani, A. Nagata, M. Muro, "Intramuscular and surface electromyogram changes during muscle fatigue”. J. Appl. Phyiol. 60(4), 1179-1185, 1986.

25. P.V. Komi, P. Tesch, "EMG frequency spectrum, muscle structure, and fatigue during dynamic contractions in man. Eur". J. Appl. Physiol. 42, 41-50, 1979.

26. H.A. DeVries, "Efficiency of electrical activity as a physiological measure of the functional state of muscle tissue". Amer J phys Med 47(1), 10-22, 1986

27. T.I. Arabadzhiev, V.G. Dimitrov, N.A. Dimitrova, et al, "Interpretation of EMG integral or RMS and estimates of neuromuscular efficiency can be misleading in fatiguing contraction". Journal of Electromyography and Kinesiology. 20(2), 223-232, 2010.

28. P. Konrad, The abc of emg. A practical introduction to kinesiological electromyography, Noraxon; 2005.

29. K. Hakkinen, M. Kallinen, M. Izquierdo, K. Jokelainen, H. Lassila, E. Malkia, et al, "Changes in agonist-antagonist EMG, muscle CSA, and force during strength training in middle-aged and older people". J Appl Physiol. 84, 1341-1349, 1998.

30. B. Carolan, E. Cafarelli, "Adaptations in coactivation after isometric resistance training". J Appl Physiol. 73, 911-917, 1992.

31. E. Hultman, H. Sjoholm, Biochemical causes of fatigue. Human muscle power, 1986. 
32. S. McMahon, D. Jenkins, "Factors affecting the rate of phosphocreatine resynthesis following intense exercise". Sports Med. 32, 761-784, 2002.

\section{AUTHORS PROFILE}

Author-1

Photo

Author-2

Photo

Author-3

Photo

Author-3

Photo

Kim, Ki-Hong, Professor, Department of Physical Education, Dankook University

Director of University Swimming Federation

Director, Korean Society of Exercise

Director, Korean Exercise Physiology Association

Director of Korean Sports Society.

Lee, Il-Suk, Professor of English at Dankook University

Jung, Song-Young, Dankook University graduate school physical education doctoral course

Yang, Chun-Ho, Professor, Dept. of Leisure Marine Sports, Hanseo University, Korea

Chief of Library, Hanseo University, Korea 